\title{
The $b$ mating-type locus of Ustilago maydis contains variable and constant regions
}

\author{
James W. Kronstad' ${ }^{\text {and Sally A. Leong }}{ }^{2}$ \\ ${ }^{1}$ Biotechnology Laboratory, Departments of Microbiology and Plant Science, University of British Columbia, Vancouver, \\ British Columbia, Canada; ${ }^{2}$ Plant Disease Resistance Unit, U.S. Department of Agriculture-Agricultural Research Service, \\ Department of Plant Pathology, University of Wisconsin, Madison, Wisconsin 53706 USA
}

The $b$ locus of the phytopathogenic fungus Ustilago maydis encodes a multiallelic recognition function that controls the ability of the fungus to form a dikaryon and complete the sexual stage of the life cycle. The $b$ locus has at least 25 alleles and any combination of two different alleles, brought together by mating between haploid cells, allows the fungus to cause disease and undergo sexual development within the plant. An open reading frame of $\mathbf{4 1 0}$ amino acids has been shown to specify a polypeptide responsible for the activity of the b1 allele, and comparisons of the predicted amino acid sequences for $6 b$ alleles allowed identification of variable and constant regions within the coding region of the gene. Haploid strains carrying a null mutation at the $b$ locus, created by gene disruption, are viable but fail to interact with formerly compatible strains to give an infectious dikaryon. Analysis of mutants carrying a null allele indicated that the products of different alleles of the $b$ locus combine to form a new regulatory activity and that this activity directly or indirectly turns on the pathway leading to sexual development and pathogenesis.

[Key Words: Recognition; plant pathogen; multiallelic gene; dikaryon]

Received April 29, 1990; revised version accepted June 8, 1990.

The basidiomycete fungus Ustilago maydis causes a disease on corn characterized by the presence of prominent tumors (galls) on the leaves, stems, ears, and tassels of the plant (Christensen 1963). Three cell types occur during the life cycle of the fungus: yeast-like haploid cells, dikaryotic cells resulting from mating between haploid strains, and diploid teliospores formed by sporulation of the dikaryon within the plant (Day and Anagnostakis 1971). Haploid cells of $U$. maydis are saprophytic and nonpathogenic; in contrast, the dikaryotic form of the fungus is difficult to culture and is generally found only in the infected plant (Day and Anagnostakis 1971). Infection of the plant, whether initiated by mating or directly from germinating teliospores, is required to complete the sexual stage of the life cycle. This is because the diploid teliospores, which germinate to give meiotic products, can only be formed in association with living plant tissue (Christensen 1963; Hausen and Beiderbeck 1987).

Mating between haploid cells is controlled by two genetic loci, the $a$ locus with two alleles, $a 1$ and $a 2$ and the $b$ locus with at least 25 alleles (Rowell 1955a,b; Holliday 1961; Puhalla 1968; Silva 1972; Day 1974). The $a$ locus appears to control cell fusion (Rowell 1955a), and the $b$ locus controls events after fusion, including establishment of the infectious dikaryon and sexual development within the plant (Rowell and DeVay 1954; Day et al.
1971). The functions of the $a$ and $b$ mating-type loci have been demonstrated with diploid strains isolated from immature gall tissue or from matings between haploid strains carrying complementary auxotrophic mutations (Rowell 1955a; Holliday 1961; Puhalla 1969). Diploid strains heterozygous at both $a$ and $b$ form mycelial colonies on nutrient medium and induce gall formation when injected into corn seedlings (Holliday 1961; Day et al. 1971). In contrast, strains homozygous at both $a$ and $b$ form yeast-like colonies and do not induce gall development (Holliday 1961; Puhalla 1969; Day et al. 1971). Diploids homozygous at $a$, but carrying any two different $b$ alleles, are pathogenic, thus demonstrating that the $b$ locus controls the formation of the infectious dikaryon (Holliday 1961; Puhalla 1968, 1970; Banuett and Herskowitz 1989). It is fascinating, given the multiallelic nature of the gene (at least 25 alleles), that each combination of alleles can be distinguished and that the state (homozygous or heterozygous) of the $b$ locus influences not only pathogenicity and sexual development but also cell morphology.

The $b$ locus presents an excellent opportunity to study a mechanism of self versus nonself recognition involving a multiallelic gene in a relatively simple organism. $U$. maydis is attractive experimentally because both classical genetic and molecular genetic techniques can be applied to the analysis of mating and pathoge- 
nicity (for review, see Froeliger and Kronstad 1990). To begin an analysis of the $b$ locus, we identified clones containing the $b 1$ and $b 2$ alleles by complementation and hybridization (Kronstad and Leong 1989). These clones were shown to encode $b$ alleles by their effect on the colony morphology and the pathogenicity of various haploid and diploid strains. Further proof came from a gene replacement experiment in which a strain carrying $a b 2$ allele was transformed with a linear DNA fragment carrying the $b 1$ allele and a selectable marker. The transformants resulting from gene replacement events carried the $b 1$ allele linked to the selectable marker. Interestingly, it was also possible to obtain haploid strains that were both mycelial and pathogenic simply through introduction of a different $b$ allele by transformation (Kronstad and Leong 1989). This result serves to confirm further the idea that the $b$ locus is the key regulatory locus controlling the ability of the fungus to cause disease on the plant.

Schulz et al. (1990) reported recently the cloning and sequence analysis of four $b$ alleles, $b 1, b 2, b 3$, and $b 4$. A single open reading frame (ORF) of 410 amino acids was found for each allele, and sequence comparisons of the alleles revealed a variable amino-terminal portion and a conserved carboxy-terminal region. The analysis of Schulz et al. (1990) also revealed that the $b$ polypeptides contain a motif related to a homeo domain suggesting that the $b$ polypeptides may be DNA-binding proteins.

In this report, we describe the localization of the $b 1$ gene on cloned DNA and the introduction of a stop codon into the $b 1$ allele to identify the correct ORF and to demonstrate that a protein product is responsible for $b$ allele activity. Sequence comparisons of six alleles confirm that the alleles are highly homologous but contain variable and constant regions. The sequence analysis reported here also allows a description of a putative nuclear localization sequence in the constant region of the $b$ alleles. In addition, we constructed a null mutation of the $b 1$ allele and introduced this mutation into haploid and diploid strains by gene replacement. Analysis of strains carrying the null mutation revealed that the $b$ locus does not encode an essential gene product and that the products of different $b$ alleles must act together to activate the pathway leading to mycelial growth and pathogenicity.

\section{Results}

\section{Location of the b1 allele on cloned DNA}

Restriction maps of the 8.5-kb BamHI fragments carrying the $b 1$ and $b 2$ alleles were constructed to assess the level of overall similarity between the alleles and to localize the alleles on cloned DNA. The 6-kb regions on the left in Figure 1 contain identical restriction sites for the nine enzymes employed to construct the maps. The $2.5-\mathrm{kb}$ regions on the right show restriction site polymorphisms for six of the nine enzymes, suggesting that the difference between the alleles might reside in this region. Overall, the restriction-site analysis revealed that the regions carrying the $b 1$ and $b 2$ alleles were quite

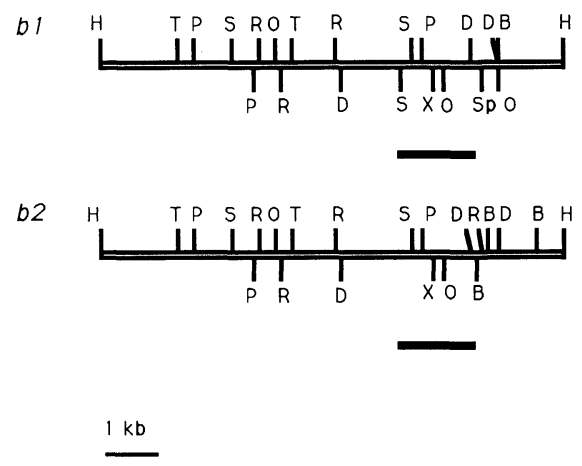

Figure 1. Restriction maps of DNA fragments carrying the $b 1$ and $b 2$ alleles. Cleavage sites for nine enzymes are shown on the 8.5-kb BamHI fragments carrying the $b 1$ and $b 2$ alleles. The cloning of these fragments was described previously (Kronstad and Leong 1989|. The positions of the ORFs for the alleles, as identified in Figs. 2 and 3, are shown as bars below the maps. The restriction enzyme designations are as follows: (B) BgIII; (D) HindIII; (H) BamHI; (S) SalI; (X) XbaI; (O) XhoI; (P) SphI; (T) PstI; (R) EcoRI; (Sp) SspI.

similar, especially in the 6-kb region containing identical restriction sites for the two alleles. This is in contrast to the situation for mating type genes from other fungi, such as Neurospora crassa, where the alleles are contained within large regions of nonhomologous DNA (Glass et al. 1988; Metzenberg and Glass 1990).

The location of the $b 1$ allele on cloned DNA was defined further by Tn 5 mutagenesis and subcloning (Fig. $2 \mathrm{~A}, \mathrm{~B})$. The activity of the $b 1$ allele was assayed by introduction of plasmids carrying the wild-type allele, subcloned fragments, or fragments with $\mathrm{Tn} 5$ insertions into a b2/b2 diploid strain (d410) and screening the transformants for the mycelial colony morphology expected of a strain carrying two different $b$ alleles. Previous studies (Kronstad and Leong 1989) have shown that the colony morphology of the strains is an accurate indication of their ability to induce galls; that is, mycelial transformants are pathogenic when injected into corn seedlings, and yeast-like strains are not. DNA fragments carrying the wild-type $b 1$ allele generally gave $70-90 \%$ mycelial transformants in this assay, as did most of the Tn5 insertions throughout the $8.5-\mathrm{kb}$ region containing the $b 1$ allele. An exception was found for the plasmid carrying $\mathrm{Tn} 5$ insertion 93; this plasmid yielded a reduced percentage of mycelial transformants (indicating disruption of b1 allele function) compared with the plasmid without Tn5. In contrast, Tn5 insertions 45 and 80, which flank 93, did not interfere with the activity of the b1 allele. It was common in these experiments, and previous ones (Kronstad and Leong 1989), to find that $<100 \%$ of the transformants with the wild-type $b$ allele showed the mycelial phenotype. It may be that some transformants integrate only part of the transforming DNA or that integration occurs at a site where the DNA is not expressed.

In addition to $\operatorname{Tn} 5$ mutagenesis, the $b 1$ allele was localized by subcloning DNA fragments into plasmid vectors (Fig. 2B) and testing the plasmids for activity 
Figure 2. Location of the $b 1$ allele on cloned DNA and effect of an in-frame stop codon. Plasmids carrying the $b 1$ allele or derivatives were assayed for $b 1$ activity by transformation into a $b 2 / b 2$ diploid strain (d410). The activity of the plasmids in this assay is indicated as the percent of the transformants forming the mycelial colony phenotype expected of a strain carrying different $b$ alleles. The restriction enzyme designations are as follows: (B) BgIII; (D) HindIII; (H) BamHI; (S) SalI; (X) XbaI. $(A)$ The 8.5-kb BamHI fragment (b1 allele), and derivatives containing insertions of transposon $\mathrm{Tn} 5$, in the cosmid vector pCU3 (Wang et al. 1989). Three Tn5 insertions $(45,80$, and 93) are shown; the exact positions of insertions 45 and 93 were determined by sequence analysis (Fig. 3), and the position of Tn5 insertion 80 was determined by restriction-site mapping. ( $B$ ) DNA fragments subcloned in the vector plC19RHL (Kronstad and Leong 1989); bl-S, 2.8-kb BamHI-Sall fragment; b1-X, 2.4-kb BamHI-XbaI fragment; bl-BS, 1.7-kb BglII-SalI fragment. $(C)$ The results of transformation with $2.8-\mathrm{kb}$ BamHI-SalI fragments carrying stop codons generated by cleaving the DNA at an upstream (bl-2) or a downstream (bl-3) HindIII restriction site and making the ends blunt with DNA polymerase (Maniatis et al. 1982). Transformation was also performed with a similar plasmid carrying a deletion of the 399-bp HindIII fragment, which removed the 5 '-flanking region and the first $30 \mathrm{bp}$ of the coding sequence. These modified fragments were cloned in plC19RHL prior to transformation into $U$. maydis.

when transformed into a $b 2 / b 2$ diploid strain (d410). The smallest fragment that gave activity, at approximately the same level as the original clone, was a 1.7-kb BglIISall fragment. A 2.5-kb BamHI-XbaI fragment, which overlapped the same region, showed a reduced frequency of mycelial transformants, indicating that part or all of the allele was missing. These experiments indicated that part or all of the $b 1$ activity was encoded by the $1.7-\mathrm{kb}$ region between the BgIII and SalI sites. The nucleotide sequence of a region extending from the BglII site to $\sim 400$ bp beyond the Sall site was determined for the $b 1$ allele. The corresponding region from the $b 2$ allele was also sequenced and, as described below, oligonucleotide primers were designed to amplify and clone equivalent sequences from four additional alleles.

\section{Nucleotide sequence of the bl allele}

Analysis of the nucleotide sequences of the $b 1$ and $b 2$ alleles for possible coding regions revealed that both had a single long ORF of 410 amino acids. The nucleotide sequence of the $b 1$ allele is shown in Figure 3. Several lines of evidence indicated that the 410-amino-acid ORF encodes a polypeptide with $b 1$ allele activity. First, transposon Tn5 insertion 45 (Figs. 2A and 3), present $189 \mathrm{bp}$ upstream of the initiation codon, did not significantly affect $b 1$ activity when transformed into a $b 2 / b 2$ diploid, whereas insertion 93 (Figs. 2A and 3), present within the ORF, did reduce $b 1$ activity. Second, the sequence at the initiation codon, CACAATGTC, matched (in 8 of 9 positions) the consensus for the sequence at fungal translation initiation codons as described by Ballance (1990).

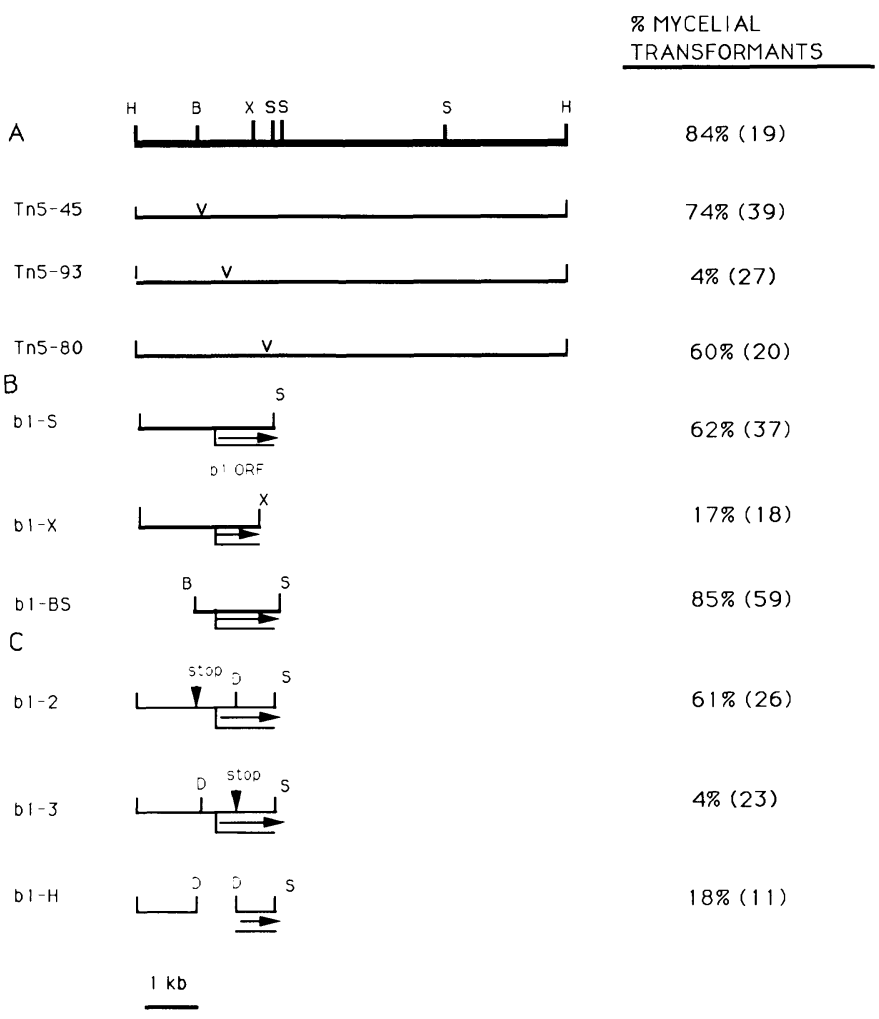

The third piece of evidence was that creation of an inframe stop codon 10 amino acids downstream (at position 444 on the nucleotide sequence) drastically reduced $b 1$ activity when transformed into a b2/b2 diploid (Fig. 2C). Deletion of a 400-bp region encompassing the initiation codon and upstream sequences also reduced $b 1$ allele activity (Fig. 2C). These results indicate that the 410-amino-acid ORF for $b 1$ encodes a polypeptide responsible for the activity of the allele. Interestingly, the ORF actually extends beyond the SalI site used to define the smallest active region (Fig. 2B). It appears, therefore, that the sequence encoding the last 78 amino acids at the carboxyl end of the $b 1$ allele can be removed without significantly reducing activity. A deletion extending to the $X b a I$ site (removing the coding sequence for 184 amino acids) significantly reduced b1 activity (Fig. 2B). A deletion that removed sequences downstream of a Sall site for the $b 2$ allele was also reported by Schulz et al. (1990). Unlike the 1.7-kb BgIII-SalI fragment and the 2.8-kb BamHI-SalI fragment from $b 1$ (b1-BS and b1-S, respectively; Fig. 2B|, the deletion in the $b 2$ allele showed a reduced level of activity when transformed into $U$. maydis. Other changes at or near this position, such as insertion of $\operatorname{Tn} 5(\operatorname{Tn} 5-80$; Fig. $2 \mathrm{~A})$ or a kanamycin marker (Schulz et al. 1990), did not reduce activity. Because the deletions and insertions removed or displaced part of the ORF, the different effects of changes in this region may be due to differences in the sequences added onto the $b$ allele coding region.

Computer analysis of the amino acid sequence of the b1 ORF indicated that the predicted polypeptide was generally hydrophilic and basic. Comparison of the 
amino acid and the nucleic acid sequences with the NBRF, EMBL, and Genbank data bases failed to identify extensive similarities, although the search did reveal a short region with sequence similarity to the nuclear localization sequence of the mouse glucocorticoid receptor (Fig. 4A; Danielsen et al. 1986; Guiochon-Mantel et al. 1989) and to histone genes (Fig. 4B; Ohe et al. 1986). This region also contains a motif similar to that described by Dingwall et al. (1989) for nuclear localization sequences; that is, a periodic motif with three basic tracts separated by two sequences of four hydrophobic residues. The $b 1$ peptide, however, has three tracts of basic amino acids separated by two stretches of either two or three hydrophobic amino acid residues (underlined in Fig. 4A). Schulz et al. (1990) also reported that searches of the NBRF and EMBL data bases failed to identify sequences with extensive similarity to the $b$ alleles; they suggested, however, that a sequence between amino acids 126 and 180 has similarity to the homeo domain found in certain Drosophila regulatory genes (Scott et al. 1989).

\section{Variable sites in the $\mathrm{b}$ locus}

Comparison of the ORFs for the $b 1$ and $b 2$ alleles revealed the presence of a variable amino-terminal region and a constant carboxyl domain (Fig. 5). Specifically, 43 amino acids in the 160 residues at the amino terminus were different between the two alleles, and only 1 residue was different in the remaining 250 residues. The latter change was the conservative substitution of aspartic acid for glutamic acid. To confirm that the variable and constant regions were general features of the $b$ alleles, similar regions were sequenced from four additional alleles. Oligonucleotide primers were designed based on the sequences of the b1 and b2 alleles (Fig. 3), and DNAs for the coding regions of four other alleles were obtained with the polymerase chain reaction (PCR). The alignments of each predicted amino acid sequence confirmed the variable and constant domain organization and also allowed further definition of the variable region (Fig. 5). The primary region of variability was between amino acids 40 and 110, with highly vari-

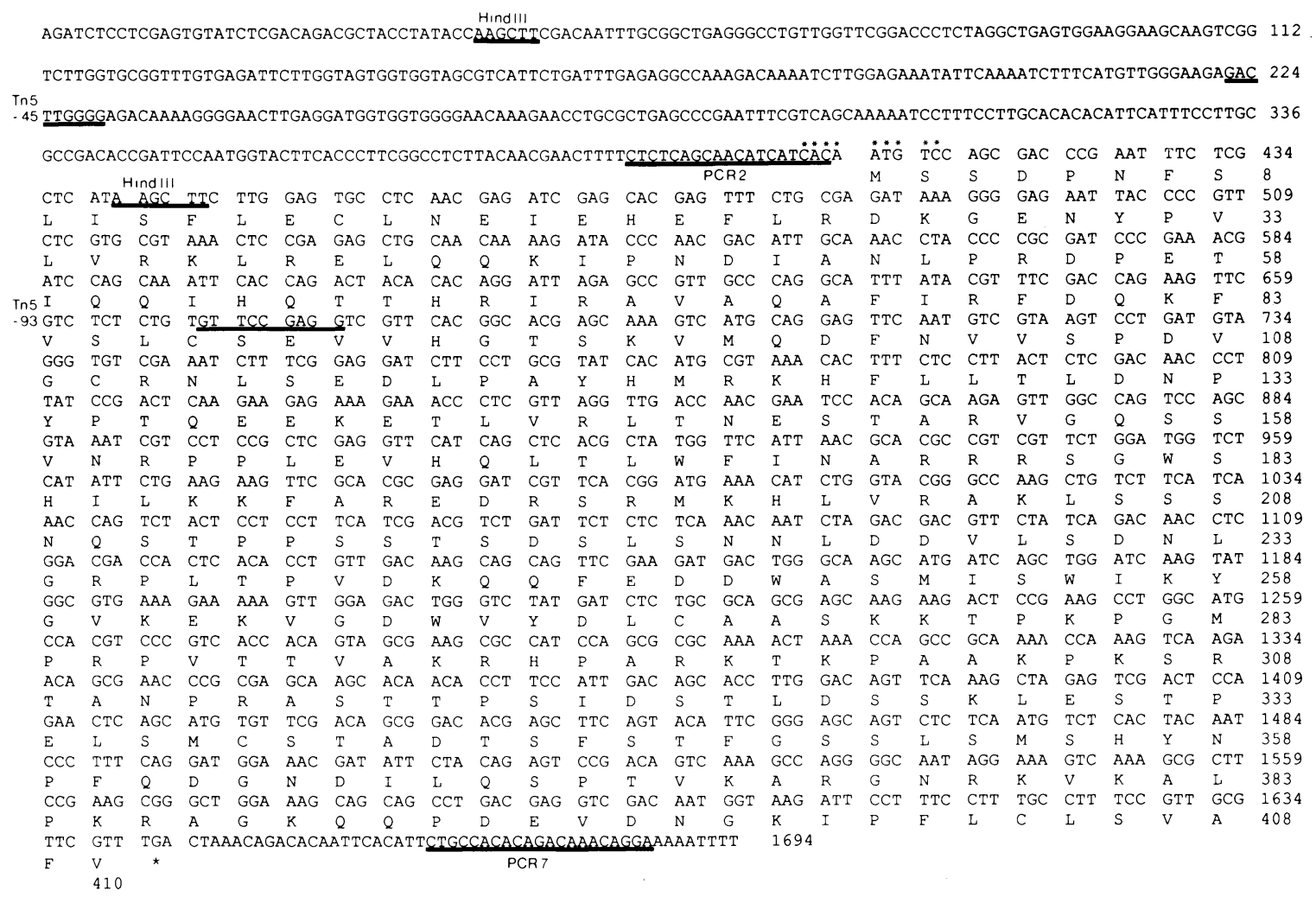

Figure 3. Nucleotide sequence of the $b 1$ allele. The nucleotide sequence of a $1.7-\mathrm{kb}$ region containing the $b 1$ allele is shown and the predicted amino acid sequence is listed using the one letter code. The positions of the HindIII sites used for generating stop codons are underlined, as are the 9-bp sequences duplicated by Tn5 insertions 45 and 93 . The positions of oligonucleotide primers PCR2 (upstream of the initiation codon) and PCR7 (downstream of the stop codon), used to amplify additional alleles, are also underlined. The sequence of PCR7 is complementary to the underlined sequence. Each of the PCR primers was synthesized to include a BamHI restriction site at the $5^{\prime}$ end so that the amplification products could be cloned as BamHI fragments. The consensus sequence for translation initiation in fungi (Ballance 1990) is marked with asterisks. The numbers on the right indicate the nucleotide and amino acid sequence positions. It should be noted that Schulz et al. (1990) have reported preliminary evidence for a 74-bp intron near the end of the ORF. The actual ORF, therefore, would encode a polypeptide of 473 amino acids for the $b 2$ allele. The carboxyl terminus of the gene, including the region containing the intron, has been shown to be dispensable for $b$ allele activity (Fig. 2B, subclones b1-S and bl-BS; Schulz et al. 1990). 
able sequences between positions 47 and 60 and between 70 and 75 . Other less striking regions of variability were present between positions 1 and 10, 28 and 31 , and 90 and 110 . Within the conserved region (111 to 410 ), two variable regions were present between residues 164 and 170 and between 216 and 221 . It should be noted that no single position contained a different amino acid in each allele; at most, four different residues were found at any single position. Alignments of different pairs of alleles also indicated that the important sequences for specifying allelic differences were located between positions 40 and 160. For example, alignment of the $b 3 / b H$ and $b 6 / b K$ alleles revealed that they are identical for the first 49 amino acids and that they show extensive sequence identity after position 160 (Fig. 5); as mentioned above, alignment of the $b 1$ and $b 2$ alleles revealed that they are also identical essentially for the carboxy 250 amino acid residues ( 1 conservative change at position 396). Of course, when other pairs of alleles are considered, differences outside the region between positions 40 and 160 may be important for allelic recognition.

The predicted amino acid sequences of the $b 1, b 2, b H$, $b J, b K$, and $b L$ alleles have been compared with the sequences of $b 1, b 2, b 3$, and $b 4$ reported by Schulz et al. (1990). The b1 alleles show differences at four amino acid positions (see Methods), the $b 2$ alleles are identical, and the $b H$ and $b 3$ alleles appear to be different isolates of the same allele (see Methods). The extent of the sequence identity for $b H$ and $b 3$ is surprising because the strains carrying these alleles were isolated independently. The $b 4, b J, b K$, and $b L$ alleles all appear to be unique; the latter three alleles have been assigned numbers $(b J=b 5, b K=b 6$, and $b L=b 7)$ to maintain a consistent nomenclature.

\section{Construction of a null mutation at the $\mathrm{b}$ locus}

Previous studies have shown that one-step gene replacement occurs at a high frequency in $U$. maydis (Kronstad et al. 1989) and that the technique can be used to perform genetic analyses (Holden et al. 1989). Therefore, we employed gene replacement to construct a null mutation in the $b 1$ allele to address two questions. First, does the $b$ locus encode an essential product, and second, if the product(s) is not essential, what is the phenotype of a haploid strain carrying a null allele? A diagram of a recombinant plasmid used to create a null mutation of the b1 allele is shown in Figure 6A. The mutation was constructed by replacement of the coding region for the first 226 amino acids of the $b 1$ ORF and 397 bp of 5'-flanking sequence with a DNA fragment specifying resistance to hygromycin B. Previously, it was found that integration of the marker specifying resistance to hygromycin B 398 bp upstream of the $b 1$ allele in the genome (at the BglII site; Fig. 1) did not affect the activity of the allele (Kronstad and Leong 1989). Therefore, it was expected that the construction shown in Figure 6A should cause a specific loss of $b 1$ allele activity.

Transformation of haploid strains [strains 518 (a2 b2) and $521(a 1 b 1)]$ with a 6.9-kb BamHI-XhoI fragment from the plasmid carrying a null mutation (Fig. 6A) yielded hygromycin B-resistant transformants with altered $b$ allele activity as assayed by mating reactions on culture medium (Fig. 6B). Haploid strains carrying different alleles at both $a$ and $b$ normally interact, when mixed on agar plates, to form colonies covered with a white, aerial mycelium; in contrast, mixtures of strains carrying the same alleles at either $a$ or $b$ form yeast-like colonies. These differences in the interactions form the basis for a plate assay to score the $a$ and $b$ alleles (Puhalla 1968). The haploid transformants presumed to contain a replacement of the genomic $b 1$ or $b 2$ alleles with the null mutation were viable on complete and minimal media but they did not react with other strains to give mycelial colonies (Fig. 6B). This result suggests that these transformants contain a null mutation at the $b$ locus. Additional evidence that the construction shown in Figure 6A results in a null mutation comes

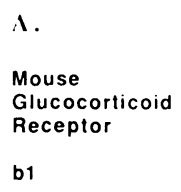

B.

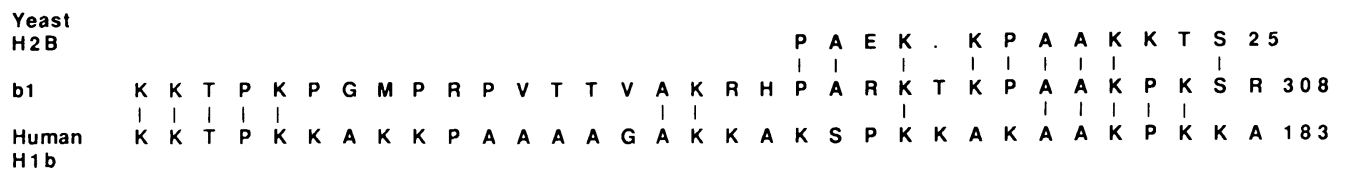

Figure 4. A potential nuclear localization sequence in the $b 1$ polypeptide. The amino acid sequence (one letter code) of the $b 1$ polypeptide between residues 276 and 308 is aligned with a similar region from $(A)$ the mouse glucocorticoid receptor (Danielsen et al. 1986) and $(B)$ two histone genes (Wallis et al. 1980; Ohe et al. 1986). The region shown for the glucocorticoid receptor is believed to play a role in nuclear localization (Guiochon-Mantel et al. 1989). The underlined part of the b1 sequence $(A)$ indicates three clusters of basic amino acid residues (separated by two clusters of hydrophobic residues); this region has a similar organization to the nuclear localization motif described by Dingwall et al. (1989) for nucleoplasmin. The region of the $b 1$ polypeptide with similarity to the histone genes contains pentapeptide motifs (beginning with proline and containing one to three basic amino acids) commonly found in histone genes and thought to play a role in the interaction between DNA and nuclear proteins (Von Holt et al. 1979). 
from the analysis of transformants of the diploid strain $\mathrm{d} 132(a 1 / a 2 \mathrm{~b} 1 / \mathrm{b} 2)$. This diploid normally forms mycelial colonies due to the presence of two different $b$ alleles; transformants were found, however, that exhibited a yeast-like colony morphology as if they had become homozygous at the $b$ locus. An example of this type of transformant is shown in Figure 6C. Because diploids of $U$. maydis are capable of participating in the mating process (Holliday 1961; Puhalla 1970), it was possible to determine which allele, $b 1$ or $b 2$, had been replaced during transformation. As shown in Figure 6C, the diploid transformant will mate with $a 1 b 1$ and $a 2 b 1$ strains but not with $a 1$ b2 or $a 2$ b2 strains, indicating that the $b_{1}$ allele has been replaced in this transformant. This result also reveals that the null allele created by gene replacement does not interfere with the interaction of the wildtype $b 1$ and $b 2$ alleles during mating.

Hybridization experiments confirmed that a direct replacement of the $b 1$ or $b 2$ allele with the disruption

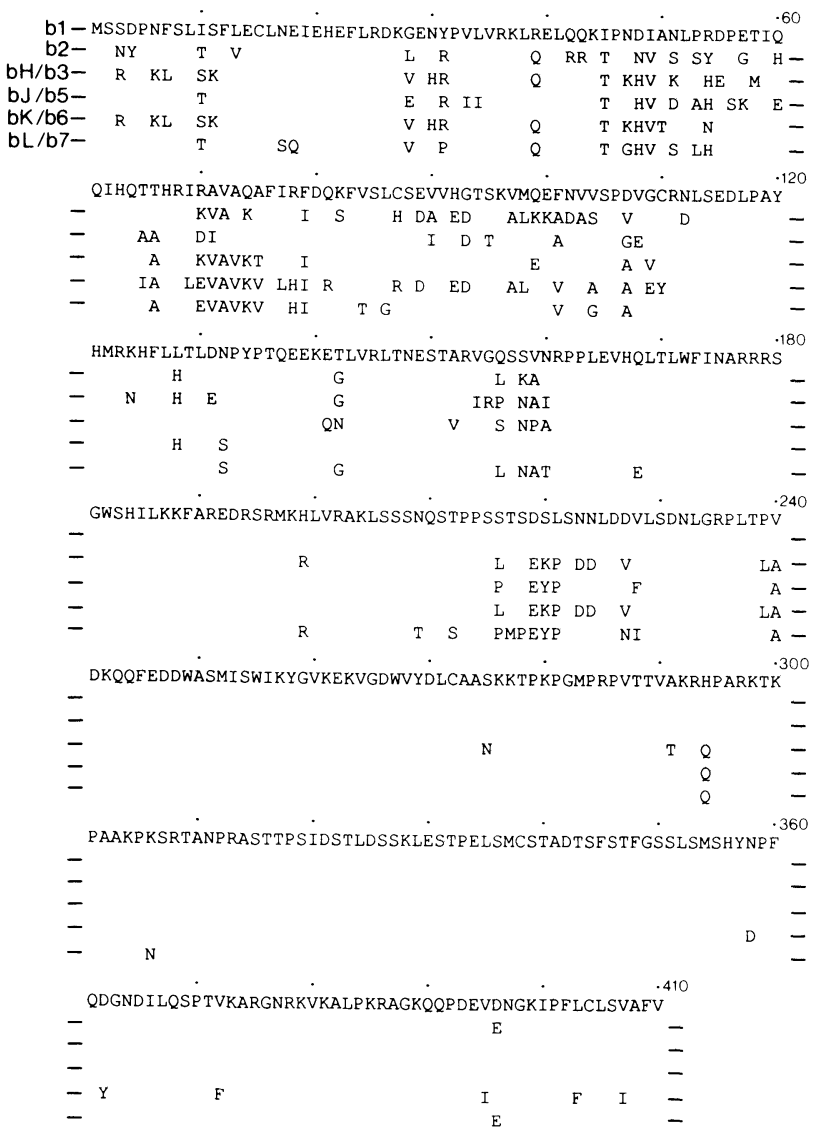

Figure 5. Alignment of $b$ allele amino acid sequences. The predicted amino acid sequences for the 410 amino acid ORFs of six $b$ alleles are shown using the one letter code. The sequences for the $b H, b J, b K$, and $b L$ alleles were predicted from the nucleotide sequence of amplified DNA cloned in pGEM3Z $\left(\mathrm{f}^{+}\right)$(see Methods); the nucleotide sequence of parts of the variable regions from these alleles was also determined directly from templates prepared by the PCR (see Methods). Note that the $b J, b K$, and $b L$ alleles have been assigned numbers $\mid b J=b 5$, $b K=b 6$, and $b L=b 7)$. fragment had occurred in the haploid strains showing an altered mating phenotype (Fig. 6D). Specifically, the 6.9$\mathrm{kb} B a m \mathrm{HI}-X h \mathrm{I}$ fragment used for transformation is present in the transformants in place of the 1.3-kb BamHI-XhoI fragment of the $b 1$ allele or the 2.1$\mathrm{kb} B a m H I-X h o I$ fragment of the $b 2$ allele. Digestion of the genomic DNA with BamHI alone revealed that the 8.5-kb BamHI fragments carrying $b 1$ or $b 2$ had been replaced, in the transformants, with the $10.2-\mathrm{kb} B a m H I$ fragments expected from a direct replacement event. In confirmation of the mating assays (Fig. 6C), hybridization analysis of the diploid transformant revealed that a direct replacement of the $b 1$ allele had occurred (Fig. $6 \mathrm{D})$; the 1.3-kb BamHI-Xhol fragment of the $b 1$ allele, but not the $2.1-\mathrm{kb}$ fragment of the $b 2$ allele, had been replaced. Digestion of genomic DNA from the diploid transformant with BamHI also demonstrated that a replacement of one of the $b$ alleles had occurred (Fig. 6D).

Overall, the transformation results demonstrate that the $b$ locus does not encode an essential gene product because haploid cells can grow, under the conditions employed, without a functional gene. As discussed below, these results also suggest that the products of two different $b$ alleles, when brought together by mating, directly or indirectly activate genes responsible for the mycelial phenotype and pathogenicity. Because the haploids carrying the null $b$ alleles did not show the mycelial phenotype by themselves, the products of the $b$ locus do not appear to repress directly the expression of genes required for the mycelial, pathogenic phenotype.

The haploid and diploid strains carrying a null allele of the $b$ locus were also tested for their ability to cause disease alone or in combination with other haploid strains (data not shown). Galls are generally obtained on corn seedlings when mixtures of compatible haploid strains, that is, strains carrying different alleles at $a$ and $b$ (e.g., $a 1 b 1+a 2 b 2)$, are injected. In no case were disease symptoms detected when haploids with a null $b$ allele were included in the plant inoculations. For example, an a1 b1:: HygB strain failed to cause disease symptoms when injected alone or when coinjected with either $a 2$ $b 2$ or $a 2 b 1:: H y g B$ strains. This finding indicates, in support of the plate mating assays, that the null mutants cannot productively interact with compatible strains to form the dikaryon. The diploid transformant /a1/a2 $b 1:: H y g B / b 2$ ) failed to give disease symptoms when injected into seedlings; in contrast, the parent strain $(a 1 / a 2 b 1 / b 2)$ incites large galls when injected alone (data not shown; Kronstad and Leong 1989).

\section{Discussion}

\section{Variable and constant regions in the $\mathrm{b}$ alleles}

The $b$ locus of $U$. maydis controls the ability of the fungus to form a stable dikaryon that can infect corn tissue and undergo sexual development. We localized the $b 1$ allele on cloned DNA by subcloning and transposon mutagenesis, and we demonstrated that a protein product is necessary for $b$ allele activity. Sequence analysis revealed ORFs of 410 amino acids for both the $b 1$ 


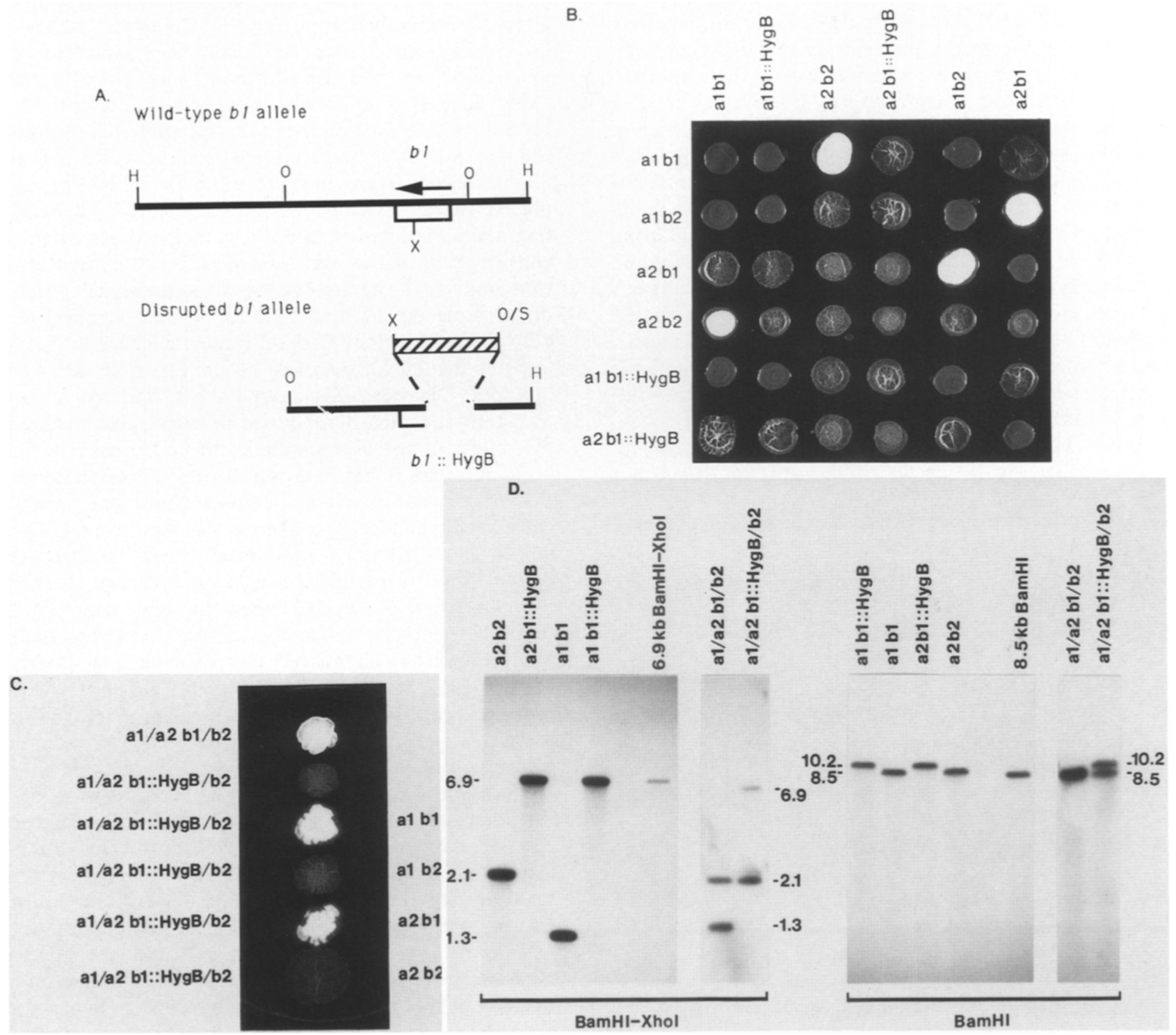

Figure 6. Disruption of the $b 1$ allele. $(A)$ The position of the $b 1$ allele and the restriction sites used for constructing a disruption vector are shown on an 8.5-kb BamHI fragment. A DNA fragment for use in a one-step gene disruption experiment was constructed by the deletion of a 1.1-kb region between $X h o I$ and $X b a I$ sites (nucleotide positions 8 to 1083 in Fig. 3) from the $b 1$ allele and insertion of a $\sim 2.7-\mathrm{kb}$ SalI-XbaI fragment (cross-hatched box) carrying a gene for resistance to hygromycin $\mathrm{B}$. The latter fragment was obtained from the $U$. maydis cloning vector pHLl (Wang et al. 1988). The 6.9-kb BamHI-XhoI fragment resulting from the construction was cleaved from the vector plC19R (Marsh et al. 1984) and used to transform haploid $U$. maydis strains 518 and 521 and diploid strain d132 to hygromycin B resistance. Restriction sites are as follows: $(\mathrm{H})$ BamHI, (X) XbaI, (O) XhoI, (S) SalI. Note that not all of the XhoI sites are shown. (B) Hygromycin B resistant transformants of haploid strains $518(a 2 b 2)$ and 521 (a1 b1) were tested for mating activity in mixing experiments with tester strains 031 (a1 b2), 032 (a2 b1), 518 (a2 b2), and 521 (a1 b1). Representative transformants with disrupted $b 1$ alleles are labeled al $b 1::$ HygB (strain 521) and $a 2 b 1::$ HygB (strain 518). Cultures of the tester strains and the transformants were spotted together on rich medium containing activated charcoal (Holliday 1974) and incubated at $30^{\circ} \mathrm{C} \mathrm{for} 48 \mathrm{hr}$. Strains carrying different alleles at both the $a$ and $b$ loci interact to give a white, mycelial colony morphology, whereas strains carrying the same alleles at either $a$ or $b$, or null alleles at $b$, give flat, yeast-like colonies. $(C)$ The colony morphologies of an $a 1 / a 2 b 1 / b 2$ diploid strain (d132) and a transformant of this strain carrying a null $b 1$ allele $(a 1 / a 2 b 1:: \mathrm{HygB} / b 2)$ grown on medium containing activated charcoal are shown. In addition, the mating reactions of the transformant in mixtures with haploid strains carrying the indicated $a$ and $b$ alleles are shown. The diploid transformant gives a mycelial reaction with $a 1 b 1$ and $a 2 b 1$ strains but not $a 1 b 2$ or $a 2 b 2$ strains indicating that both $a$ alleles, but only the $b 2$ allele, are present. A dual mating reaction for diploids carrying both $a$ alleles has been described previously (Holliday 1961; Puhalla 1970). (D) DNA hybridization analysis of the transformants carrying a null $b 1$ allele. Genomic DNAs from haploid strains $518(a 2$ b2) and $521(a 1 b 1)$ and diploid strain d132 and transformants of these strains were digested with both $B a m H I$ and $X h o I$ or with $B a m H I$ alone as indicated. A $1.5-\mathrm{kb} \mathrm{BamHI-XhoI} \mathrm{fragment} \mathrm{from} \mathrm{the} b 1$ allele (at the right end of the map in Fig. 1) was used as a hybridization probe. The 6.9-kb BamHI-XhoI fragment from the disruption plasmid $(A)$ and the $8.5-\mathrm{kb}$ BamHI fragment from the $b 2$ allele were loaded on the gels as markers. The numbers in the margins indicate size in kilobases. 
and $b 2$ alleles. The nucleotide sequences of four additional alleles were determined, and comparisons of the predicted amino acid sequences for the six alleles revealed the presence of variable and conserved regions. In general, the alleles possess variable amino-terminal regions of $\sim 110$ amino acids and conserved carboxy-terminal regions of 300 amino acids. Amino acid differences in the variable region occurred primarily between positions 40 and 110 with the main clusters between positions 47 and 60 and between positions 70 and 75 . Within the conserved portion, two short regions between positions 164 and 170 and between 216 and 221 showed some variability in amino acid sequence. The identification of variable and constant regions within the $b$ locus suggests experiments to test the importance of these regions for allelic specificity and for the activity of the polypeptides. Specifically, the roles of various portions of the polypeptides encoded by the $b$ alleles could be explored by altering the amino acids at the hypervariable positions in vitro and by constructing deletion derivatives of alleles (e.g., lacking the variable region) and recombinants between alleles. It may be possible, for example, to create a constitutive $b$ allele by deleting part or all of the variable portion of the gene.

\section{Comparisons with other multiallelic recognition systems}

The comparison of several $b$ alleles indicates that the $b$ locus is organized in a similar fashion to the $S$ locus of flowering plants; alleles of this gene have hypervariable regions interspersed within a framework of constant regions (Anderson et al. 1989). The $S$ locus can have hundreds of alleles in some plant species and, in the case of Nicotiana alata, the alleles show sequence similarity to ribonucleases (McClure et al. 1989). The homology found between different alleles of the $b$ locus sets this gene apart from the $A \alpha$ incompatibility locus of another basidiomycete fungus, Schizophyllum commune. In $S$. commune, a wood-rotting fungus, there are four multiallelic, incompatibility genes, $A \alpha, A \beta, B \alpha$, and $B \beta$, and monokaryons must carry different alleles at either $A \alpha$ or $A \beta$ and at either $B \alpha$ or $B \beta$ to form a dikaryon (Raper 1983). Therefore, these genes appear to perform a recognition function similar to that of the $b$ locus in $U$. maydis. It is curious, however, that two cloned $\mathrm{A} \alpha$ alleles do not cross-hybridize with each other or with genomic DNA carrying any of the other seven alleles (Giasson et al. 1989). Metzenberg and Glass (1990) proposed the term idiomorph to describe sequences, like those of the A $\alpha$ genes of $S$. commune and the A and $a$ mating type genes of Neurospora crassa (Glass et al. 1989|, that occupy the same genetic locus but are not related in sequence. Our results demonstrate that the alleles of the $b$ locus of $U$. maydis are not idiomorphs because they are highly homologous.

\section{Null mutation at the $\mathrm{b}$ locus}

Our analysis of a $b$ allele containing a null mutation suggests that the $b$ locus functions in a seemingly posi- tive manner; that is, it directly or indirectly activates the expression of genes required for pathogenesis in strains carrying two different alleles. The reasoning is as follows. If the product of $a b$ allele functions in a haploid strain to prevent the expression of genes necessary for the mycelial phenotype and pathogenicity, then the loss of $b$ allele activity (as a result of gene disruption) should give a mycelial, pathogenic haploid. Disruption of the $b$ locus does not result in this phenotype. On the contrary, a haploid strain without a functional $b$ allele forms yeast-like colonies and fails to give the mycelial reaction indicative of dikaryon formation when mixed with a compatible strain. Therefore, it seems reasonable to postulate that when the products of two different alleles are brought together during mating, an activity is created that directly or indirectly turns on the pathway leading to mycelial growth and the ability to complete sexual development in maize. It is not simply the case that haploids are incapable of displaying the mycelial phenotype because we have shown previously that haploid transformants carrying two different $b$ alleles are both mycelial and pathogenic (Kronstad and Leong 1989). Precedent for the creation of a new regulatory activity after mating comes from the yeast Saccharomyces cerevisiae, in which products from each of the MAT alleles, a1 from MATa and $\alpha 2$ from $M A T \alpha$, combine to give a repressor $(\mathbf{a} 1-\alpha 2)$ found in diploid, but not haploid, cells. The a1 $-\alpha 2$ repressor functions to turn off the expression of haploid specific genes (Goutte and Johnson 1988) including the RME1 gene product, a repressor of sporulation and meiosis (Herskowitz 1988). A similar situation may occur for the products of the $b$ alleles except that the new activity would be created by the interaction of polypeptides encoded by different alleles of the same gene. The phenotype of a mutant carrying a null allele suggests that the activity would be positive, although, like the al- $\alpha 2$ repressor of yeast, the $b$ polypeptides could act as a repressor and turn off the expression of other regulatory genes (e.g., a repressor of pathogenicity). The analysis of the strains carrying a null allele at the $b$ locus also indicates that the alleles do not encode essential gene products.

\section{A putative nuclear localization sequence in the $\mathrm{b}$ polypeptides}

Clues about the activity of the polypeptides have come, not only from our analysis of a null mutation at the $b$ locus, but also from the sequence analysis of the alleles. Although data base searches failed to reveal extensive similarities to other genes, we did find that the polypeptide for the $b 1$ allele (and the other alleles) has a region with sequence similarity to the nuclear localization sequences of the glucocorticoid receptor (Danielsen et al. 1986; Guiochon-Mantel et al. 1989) and nucleoplasmin (Dingwall et al. 1989). The same basic stretch of amino acids in the $b$ polypeptides also shows similarity to regions of histone genes rich in proline, lysine, and alanine. The latter similarity may simply reflect the basic nature of this region of the $b$ protein; 13 of the 33 
amino acids in this region (Fig. 4) are basic and 14 are hydrophobic including 7 proline residues. The sequence similarity raises the possibility, however, that the $b$ polypeptides reside in the nucleus.

Schulz et al. (1990) have suggested, based on comparisons with Drosophila and yeast genes, that a homeo domain-like motif is present in $b$ allele polypeptides. The sequence similarity to the Drosophila antennapedia gene includes 17 matches in a region of 41 amino acids; the matches include four of the invariant residues and six of the eight highly conserved residues of the homeo domain proteins (Schulz et al. 1990). The region of sequence similarity spans the helix-turn-helix motif thought to be a DNA-recognition helix for homeo domain proteins. Most of the matches to the conserved residues of the helix-turn-helix motif of the antennapedia gene are found in the four alleles sequenced by Schulz et al. (1990), b1, b2, b3, and b4, as well as in the additional alleles described here, $b 5 / b J, b 6 / b K$, and $b 7 / b L$. All seven of the $b$ alleles sequenced to date contain the four highly conserved amino acids WF-N-R found in the homeo domain proteins (Scott et al. 1989). Certainly, a nuclear location for the $b$ polypeptides is consistent with the presence of a homeo domain-like motif since all homeo domain-containing proteins localized so far are found in the nucleus (Scott et al. 1989).

\section{Models for $\mathrm{b}$ allele function}

Schulz et al. (1990) have proposed two models for how polypeptides encoded by different $b$ alleles might interact to control formation of the dikaryon. In both models, they assume that the formation of a multimeric species (e.g., homodimers or heterodimers) is important for the function of the $b$ locus. In this respect, the models are similar to the subunit aggregation model proposed for the multiallelic recognition system of $S$. commune (Kuhn and Parag 1972). In the first model of Schulz et al. (1990), the variable region is postulated to control the association of monomeric polypeptides and the activity of the multimeric products. It is formally possible that either homodimers or heterodimers could be functional, although the fact that homodimers would theoretically be present even when a cell carries different alleles suggests that the heterodimer is the active species. As described above, our analysis of strains carrying a null mutation of the $b 1$ allele indicates that the products of different alleles interact to form a new regulatory activity; that is, the heterodimer is the active species. In their second model, Schulz et al. (1990) postulate that the constant region, rather than the variable region, governs the association of polypeptides to form multimers and that the variable region controls the activity of the multimeric species. Thus, differences in the activities of heterodimers versus homodimers would depend on the variable regions; for example, the variable regions would control the formation of recognition sites for DNA sequences upstream of target genes or for association with an accessory protein.

Our finding of a similarity between a basic region of the $b 1$ polypeptide and nuclear localization sequences for nucleoplasmin and the glucocorticoid receptor led us to consider the possibility that regulation of the intracellular location of the $b$ allele polypeptides plays a role in their activity. In general, genes whose products are subject to regulated nuclear entry contain a patch of basic amino acids thought to act as a nuclear localization signal (Hunt 1989). In a model based on regulated nuclear entry (Fig. 7), the interaction of both like and unlike $b$ polypeptides could yield a functional species, but one species (i.e., the homodimer) may be retained in the cytoplasm, due to the configuration of the variable regions, whereas the other species (i.e., the heterodimer) may be free to enter the nucleus. It is possible that the variable regions govern interaction with a protein responsible for localizing the products of the $b$ alleles to the cytoplasm or, alternatively, heterodimers alone could possess a conformation or structure that allows nuclear entry, whether or not interaction with another factor is involved. A hypothesized cytoplasmic anchoring protein would presumably distinguish between homodimers and heterodimers by conformational differences determined by the variable regions. The anchor would recognize a structure or conformation that each of the 25 types of homodimers (from 25 alleles) would be able to form; perhaps heterodimer formation need only perturb this structure to obviate recognition and anchoring. If a cytoplasmic anchoring protein does interact with the products of the $b$ alleles, then one test of the

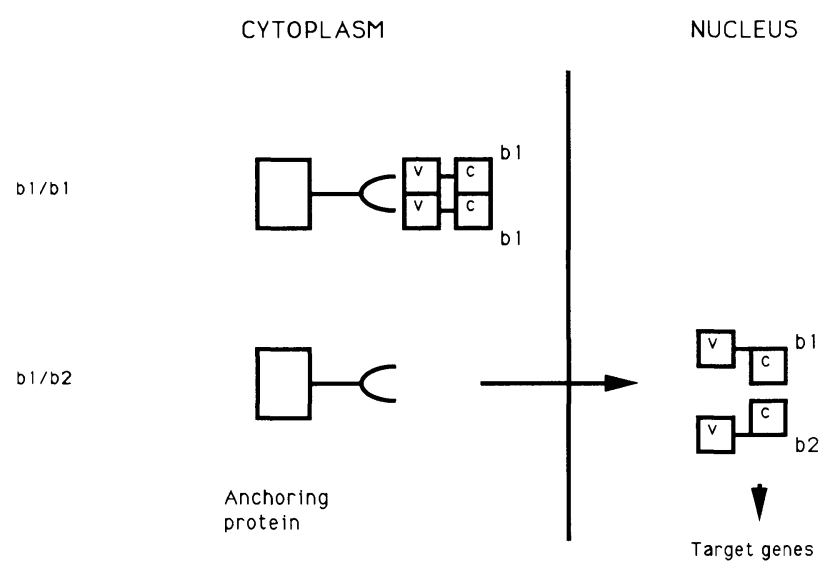

Figure 7. Model for $b$ allele function based on regulated nuclear entry. The products of the $b$ alleles are depicted as dimers with the variable $(\mathrm{v})$ and constant $(\mathrm{c})$ regions indicated as boxes. A hypothetical anchoring protein (large box in cytoplasm) is shown controlling the access of the $b$ allele dimers to the nucleus. It could also be possible, however, that the association of subunits alone (heterodimer versus homodimer) is sufficient to determine the location of the products in the cell. A conformational change of the multimer is depicted as obviating localization to the cytoplasm. Once in the nucleus, the heterodimer is depicted as influencing the expression of target genes; these could be genes encoding transcriptional activators or repressors or genes whose expression is required for the mycelial phenotype and growth of the fungus within the plant. The interactions of the constant regions would presumably not require the carboxyl terminus (i.e., the last 78 amino acids) because deletion of this region does not affect significantly activity (Fig. 2; Schulz et al. 1990). 
model would be to overexpress a $b$ allele, beyond the capacity of the anchoring protein to bind all multimers, and see if the mycelial phenotype results. Evidence for the interaction of other factors with the $b$ polypeptides might also be obtained by looking for haploid mutants that possess a mycelial phenotype but carry mutations in genes other than the $b$ alleles.

Hunt (1989) has recently reviewed a number of examples of gene products displaying regulated nuclear localization including the dorsal gene of Drosophila, the steroid receptors, and the transcriptional activator NF- $\mathrm{kB}$. A patch of basic amino acids has been described for each gene product, and candidates for the cytoplasmic anchors have been identified. In the case of the glucocorticoid receptor, association with a heat-shock protein, HSP90, appears to play a role in the localization of the receptor (Picard et al. 1988). The model proposed by Hunt (1989) for regulated nuclear entry postulates that relief from cytoplasmic anchoring is achieved by modification of the anchoring protein or of the anchored protein (e.g., by phosphorylation). Presumably, a conformational change results from the modification. As mentioned above, a model based on regulated nuclear entry of the $b$ alleles (Fig. 7) would postulate that the equivalent conformational change results from the interactions of the products of different alleles. Such a model would also be compatible with the models proposed by Schulz et al. (1990). In each case, the activity of the $b$ polypeptides or the location of the activity would depend on differences in the interactions of like and unlike products of the $b$ alleles.

\section{Summary}

It is clear that the characterization of the $b$ locus presents an excellent opportunity to elucidate a mechanism of multiallelic recognition. It is fortuitous that, in $U$. maydis, both molecular and genetic approaches can be applied to the problem. Future experiments to test the selective localization model and the other models for $b$ locus function will address questions about the location of the polypeptides in the cell and the interactions of $b$ polypeptides with each other, with other proteins, and with DNA. In addition, the central role that the $b$ locus plays in the life cycle of $U$. maydis also presents a chance to identify other genes directly or indirectly regulated by the state of the $b$ locus and to explore not only the regulation but also the mechanisms of fungal pathogenicity.

\footnotetext{
Methods

Strains and media

Escherichia coli strain $\mathrm{DH} 5 \alpha\left[\mathrm{F}^{-}\right.$, endA1, hsdR17 $\left(\mathrm{r}_{\mathrm{k}}{ }^{-}, \mathrm{m}_{\mathrm{k}}{ }^{+}\right)$, supE44, thi- ${ }^{-}$recA1, gyrA96, relA1, ф80dlacZM15] was used for all DNA manipulations. The $U$. maydis strains employed are listed in Table 1 . The strains with ATCC numbers were obtained from the American Type Culture Collection, Rockville, Maryland. Media for the culture of $U$. maydis have been described previously (Holliday 1974).
}

Table 1. U. maydis strains

\begin{tabular}{ll}
\hline Strain & Genotype \\
\hline 518 & $a 2 b 2$ \\
521 & $a 1 b 1$ \\
UM031 & $a 1 b 2$ \\
UM032 & $a 2 b 1$ \\
d410 & $a 1 / a 2 b 2 / b 2$ \\
d132 & $a 1 / a 2 b 1 / b 2$ \\
ATCC18604 & $a 1 b H(b 3)$ \\
ATCC 22907 & $a 2 b J(b 5)$ \\
ATCC 22505 & $a 1 b K(b 6)$ \\
ATCC 22891 & $a 1 b L(b 7)$ \\
\hline
\end{tabular}

Haploid strains 518 and 521 and diploid strain d132 were obtained from R. Holliday, and strains UM031 and UM032 were meiotic products of a cross between 518 and 521. The diploid strain $\mathrm{d} 410$ was constructed as described previously (Kronstad and Leong 1989a) and the other strains were obtained from the American Type Culture Collection. The mating interactions of the ATCC strains have been described (Puhalla 1970) and the $b$ allele designations changed as indicated.

\section{DNA procedures}

Nucleotide sequence analysis was performed with the Sequenase kit (U.S. Biochemicals). Fragments to be sequenced were subcloned in the vector pGEM3Z $\left(\mathrm{f}^{+}\right)$(Promega). Sequencing with double-stranded templates was performed by use of the method of Toneguzzo et al. (1988). PCR amplifications were performed with the GeneAmp reagent kit and the Thermal Cycler supplied by Perkin Elmer-Cetus. The amplification conditions were as recommended by the manufacturer except that the primers were used at a concentration of $0.2 \mu \mathrm{M}$. The amplification protocol was as follows: a $94^{\circ} \mathrm{C}$ preincubation was performed for $7 \mathrm{~min}$, followed by 25 amplification cycles $\left(94^{\circ} \mathrm{C}\right.$ denaturation, $1 \mathrm{~min} ; 55^{\circ} \mathrm{C}$ annealing, $2 \mathrm{~min} ; 72^{\circ} \mathrm{C}$ extension, $3 \mathrm{~min}$ ) and a $72^{\circ} \mathrm{C}$ final extension for $6 \mathrm{~min}$. Vectors for subcloning the $b$ alleles were pHL1 (Wang et al. 1988) and plC19RHL (Kronstad and Leong 1989); both plasmids contain an $E$. coli gene specifying resistance to hygromycin $\mathrm{B}$. This gene functions in $U$. maydis because of a transcriptional fusion with a $U$. maydis hsp 70 gene. Introduction of these vectors and derivatives into $U$. maydis by the method of Wang et al. (1988) yields transformants with integrated copies of the plasmids at a frequency of 10-50 per $\mu \mathrm{g}$ of DNA. Transformation of $E$. coli, isolation of plasmid DNA, and other DNA manipulations were performed as described (Maniatis et al. 1982). Mutagenesis with transposon Tn5 was performed as described (de Bruijn and Lupski 1984).

Transfer of DNA and hybridization to nitrocellulose filters (Sigma) were performed as described by Maniatis et al. (1982) except that formamide was present at a concentration of $40 \%$ in the hybridization buffer. [ $\left.{ }^{32} \mathrm{P}\right] \mathrm{dCTP}$ was incorporated into DNA fragments using the Random Primers Labeling System (Bethesda Research Laboratories). Total genomic DNA was isolated from $U$. maydis as described previously (Kronstad et al. 1989).

\section{Analysis of transformants}

Transformants were transferred from the primary transformation plates (Wang et al. 1988) to rich medium containing activated charcoal (Holliday 1974) and incubated at $30^{\circ} \mathrm{C}$ for two to three days. The phenotypes of the resulting colonies, whether yeast-like or mycelial, were recorded, and the transformants 
transferred to a second set of petri plates containing rich medium with activated charcoal and hygromycin B. After incubation, the phenotypes of the colonies were again recorded. The inclusion of hygromycin B in the medium for the second set of assay plates ensured that only the phenotypes of actual transformants were recorded.

\section{Sequence analysis}

DNA sequence data were analyzed by use of the software of the University of Wisconsin Genetics Computer Group (Devereux et al. 1984). Data base searches were performed with the WORDSEARCH and TFASTA programs and sequence alignments employed the PUBLISH, LINEUP and GAP programs.

Comparison of the nucleotide and predicted amino acid sequences for the $b 1, b 2, b H, b J, b K$, and $b L$ alleles with the sequences of the $b 1, b 2, b 3$, and $b 4$ alleles reported by Schulz et al. (1990) revealed that the $b 3$ and $b H$ alleles have identical amino acid sequences. However, comparison of the nucleotide sequences of $b 3$ and $b H$ revealed a 2-bp difference in codon 194. The nucleotide sequence presented by Schulz et al. (1990) would predict proline at position 194 although their amino acid sequence for $b 3$ lists arginine at this position. Therefore, it is possible that a single amino acid difference may exist between $b 3$ and $b H$; a small number of differences might be expected because these appear to be different isolates of the same allele. Comparisons of the sequence of $b 1$ with that obtained by Schulz et al. (1990) revealed amino acid discrepancies at positions $168,169,218$, and 292 . The nucleotide sequence for these codons was rechecked and found to be correct; in addition, two of the four amino acid positions are conserved completely among the six alleles and the other two are conserved in five of the six alleles. These differences may exist in the different clones of the $b 1$ allele, although they come from the same strain (521).

\section{Inoculation of corn seedlings}

Seven-day-old "Golden Bantam" (Buckerfield Seed Co., Vancouver, B.C., Canada) corn seedlings were grown in Cornell Mix and injected at the soil line with $20-50 \mu \mathrm{l}$ of fungal cell suspensions $\left(10^{7}\right.$ per $\left.\mathrm{ml}\right)$ with a $1-\mathrm{ml}$ syringe and a 26 -gauge needle. Plants were grown on a laboratory bench at $24^{\circ} \mathrm{C}$ under a bank of 8 Vitalights (DURO-TEST, Inc.).

\section{Acknowledgments}

We thank W. Benton Gibbard for help with the sequence analysis. Irv Edelman of the Wisconsin Genetics Computer Group and Don Hinkelman of the Cancer Control Agency of British Columbia provided assistance with the data base searches. We also thank Tom Atkinson for oligonucleotide synthesis, and Eunice Froeliger, Carl Douglas and N. Louise Glass for reading the manuscript. This work was supported by a Natural Sciences and Engineering Research Council operating grant to J.W.K. and by the U.S. Department of Agriculture.

The publication costs of this article were defrayed in part by payment of page charges. This article must therefore be hereby marked "advertisement" in accordance with 18 USC section 1734 solely to indicate this fact.

\section{Note added in proof}

Sequence data described in this paper have been submitted to the EMBL/GenBank Data Libraries under accession numbers X54069 (b5), X54070 (b7), and X54071 (b6).

\section{References}

Anderson, M.A., G.I. McFadden, R. Bernatzky, A. Atkinson, T. Orpin, H. Dedman, G. Tregear, R. Fernley, and A.E. Clarke. 1989. Sequence variability of three alleles of the self-incompatibility gene of Nicotiana alata. The Plant Cell 1: 483491.

Ballance, D.J. 1990. Transformation systems for filamentous fungi and an overview of fungal gene structure. In Molecular industrial mycology: Systems and applications for filamentous fungi (ed. S.A. Leong and R. Berka). Marcel Dekker, New York. (in press).

Banuett, F. and I. Herskowitz. 1989. Different $a$ alleles of Ustilago maydis are necessary for maintenance of filamentous growth but not for meiosis. Proc. Natl. Acad. Sci. 86: 58785882.

Christensen, J.J. 1963. Corn smut caused by Ustilago maydis. In American Phytopathology Society Monograph No. 2, pp. 1-41. American Phytopath. Soc., Saint Paul.

Danielsen, M., J.P. Northrop, and G.M. Ringold. 1986. The mouse glucocorticoid receptor: Mapping of functional domains by cloning, sequencing, and expression of wild-type and mutant receptor proteins. EMBO J. 5: 2513-2522.

Day, P.R. 1974. Genetics of Pathogenicity. In Genetics of hostparasite interactions (ed. A. Kelman and L. Sequeira), pp 36-91. W.H. Freeman and Co., San Francisco.

Day, P.R. and S.L. Anagnostakis. 1971. Corn smut dikaryon in culture. Nature New Biol. 231: 19-20.

Day, P.R., S.L. Anagnostakis, and J.E. Puhalla. 1971. Pathogenicity resulting from mutation at the $b$ locus of Ustilago maydis. Proc. Natl. Acad. Sci. 68: 533-535.

Devereux, J., P. Haeberli, and O. Smithies. 1984. A comprehensive set of sequence analysis programs for the VAX. Nucleic Acids Res. 12: 387-395.

Dingwall, C., J. Robbins and S.M. Dilworth. 1989. Characterization of the nuclear location sequence of Xenopus nucleoplasmin. J. Cell Sci. (Suppl.) 11: 243-248.

de Bruijn, F.J. and J.R. Lupski. 1984. The use of transposon Tn5 in the rapid generation of correlated physical and genetic maps of DNA segments cloned into multicopy plasmids-a review. Gene 27: 131-149.

Froeliger, E.H. and J.W. Kronstad. 1990. Mating and pathogenicity in Ustilago maydis. In Seminars in developmental biology: Fungal development and mating interactions (ed. C.A. Raper and D.I. Johnson). Saunders Scientific Publications, London. (in press).

Giasson, L., C.A. Specht, C. Milgrim, C.P. Novotny, and R.C. Ullrich. 1989. Cloning and comparison of A $\alpha$ mating-type alleles of the Basidiomycete Schizophyllum commune. Mol. Gen. Genet. 218: 72-77.

Glass, N.L., S.J. Vollmer, C. Staben, J. Groteluschen, R.L. Metzenberg, and C. Yanofsky. 1988. DNAs of the two matingtype alleles of Neurospora crassa are highly dissimilar. Science 241: 570-573.

Goutte, C. and A.D. Johnson. 1988. al protein alters the DNAbinding specificity of $\alpha 2$ repressor. Cell 52: 875-882.

Guiochon-Mantel, A., H. Loosfelt, P. Lescop, S. Sar, M. Atger, M. Perrot-Applanat, and E. Milgrom. 1989. Mechanisms of nuclear localization of the progesterone receptor: Evidence for interaction between monomers. Cell 57: 1147-1154.

Hausen, G. and R. Beiderbeck. 1987. The development of an in vitro host-pathogen system consisting of maize seedling segments infected with Ustilago maydis. I. Phytopathol. 118: $97-102$.

Herskowitz, I. 1988. Life cycle of the budding yeast Saccharomyces cerevisiae. Microbiol. Rev. 52: 536-553. 
Holden, D.W., J.W. Kronstad, and S.A. Leong. 1989. Mutation in a heat-regulated hsp70 gene of Ustilago maydis. EMBO I. 8: $1927-1934$

Holliday, R. 1961. Induced mitotic crossing-over in Ustilago maydis. Genet. Res. 2: 231-248.

- 1974. Ustilago maydis. In Handbook of genetics (ed. R.C. King), vol. 1, pp. 575-595. Plenum, New York.

Hunt, T. 1989. Cytoplasmic anchoring proteins and the control of nuclear localization. Cell 59: 949-951.

Kronstad, J.W., and S.A. Leong. 1989. Isolation of two alleles of the $b$ locus of Ustilago maydis. Proc. Natl. Acad. Sci. 86: $978-982$.

Kronstad, J.W., J. Wang, S.F. Covert, D.W. Holden, G.S. McKnight, S.A. Leong. 1989. Isolation of metabolic genes and demonstration of gene disruption in the phytopathogenic fungus Ustilago maydis. Gene 79: 97-106.

Kuhn, J. and Y. Parag. 1972. Protein-subunit aggregation model for self-incompatibility in higher fungi. J. Theor. Biol. 35: 77-91.

Maniatis, T., E.F. Fritsch, and J. Sambrook. 1982. Molecular cloning: A laboratory manual. Cold Spring Harbor Laboratory Press, Cold Spring Harbor, New York.

Marsh, J.L., M. Erfle, and E.J. Wykes. 1984. The plC plasmid and phage vectors with versatile cloning sites for recombinant selection by insertional inactivation. Gene 32: 481485.

Metzenberg, R.L. and N.L. Glass. 1990. Mating type and mating strategies in Neurospora. BioEssays 12: 53-59.

McClure, B.A., V. Haring, P.R. Ebert, M.A. Anderson, R.J. Simpson, F. Sakiyama, and A.E. Clarke. 1989. Style self-incompatibility gene products of Nicotiana alata are ribonucleases. Nature 342: 955-957.

Ohe, Y., H. Hayashi, and K. Iwai. 1986. Human spleen histone H1. Isolation and amino acid sequence of a main variant. $J$. Biochem. 100: 359-368.

Picard, D., S.J. Salser, and K.R. Yamamoto. 1988. A movable and regulable inactivation function within the steroid binding domain of the glucocorticoid receptor. Cell 54: $1073-1080$.

Puhalla, J.E. 1968. Compatibility reactions on solid medium and interstrain inhibition in Ustilago maydis. Genetics 60: $461-474$.

1969. The formation of diploids of Ustilago maydis on agar medium. Phytopathol. 59: 1771-1772.

. 1970. Genetic studies of the $b$ incompatibility locus of Ustilago maydis. Genet. Res. 16: 229-232.

Raper, C.A. 1983. Controls for development and differentiation of the dikaryon in Basidiomycetes. In Secondary metabolism and differentiation in fungi (ed. J.W. Bennett and A. Ciegler), pp. 195-238, Marcel Dekker, New York.

Rowell, J.B. 1955a. Functional role of compatibility factors and an in vitro test for sexual compatibility with haploid lines of Ustilago zeae. Phytopathol. 45: 370-374.

- 1955b. Segregation of sex factors in a diploid line of $U s$ tilago zeae induced by alpha radiation. Science 121: 304306.

Rowell, J.B. and J.F. DeVay. 1954. Genetics of Ustilago zeae in relation to basic problems of its pathogenicity. Phytopathol. 44: 356-362.

Schulz, B., F. Banuett, M. Dahl, R. Schlesinger, W. Schafer, T. Martin, I. Herskowitz, and R. Kahmann. 1990. The $b$ alleles of $U$. maydis, whose combinations program pathogenic development, code for polypeptides containing a homeodomain-related motif. Cell 60: 295-306.

Scott, M.P., J.W. Tamkun, and G.W. Hartzell III. 1989. The structure and function of the homeodomain. Biochem.
Biophys. Acta. 989: 25-48.

Silva, J. 1972. Alleles at the $b$ incompatibility locus in Polish and North American populations of Ustilago maydis (DC) Corda. Physiol. Plant Pathol. 2: 333-337.

Toneguzzo, F., S. Glynn, E. Levi, S. Mjolsness, and A. Hayday. 1988. Use of a chemically modified T7 DNA polymerase for manual and automated sequencing of supercoiled DNA. BioTechniques 6: 460-469.

Von Holt, C., W.N. Strickland, W.F. Brandt, and M.S. Strickland. 1979. More histone structures. FEBS Letters 100: 201218.

Wallis, J.W., L. Hereford, and M. Grunstein. 1980. Histone H2B genes of yeast encode two different proteins. Cell 22: 799805.

Wang, J., D.W. Holden, and S.A. Leong. 1988. Gene transfer system for the phytopathogenic fungus Ustilago maydis. Proc. Natl. Acad. Sci. 85: 865-869.

Wang, J., A.D. Budde, and S.A. Leong. 1989. Analysis of ferrichrome biosynthesis in the phytopathogenic fungus Ustilago maydis: Cloning of an ornithine- $\mathrm{N}^{5}$-oxygenase gene. $J$. Bacteriol. 171: 2811-2818. 


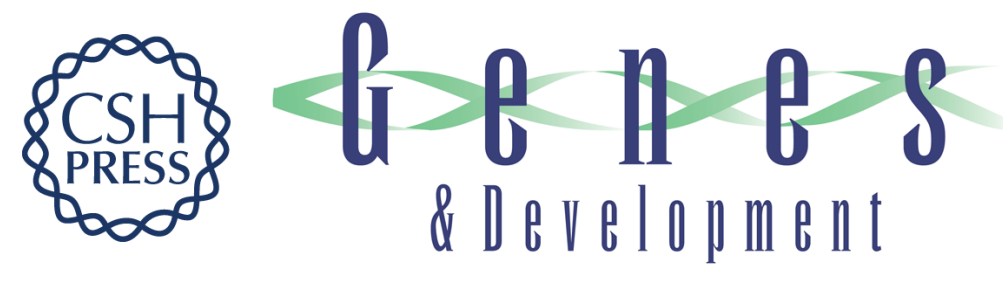

\section{The $\mathbf{b}$ mating-type locus of Ustilago maydis contains variable and constant regions.}

J W Kronstad and S A Leong

Genes Dev. 1990, 4:

Access the most recent version at doi:10.1101/gad.4.8.1384

References This article cites 39 articles, 10 of which can be accessed free at: http://genesdev.cshlp.org/content/4/8/1384.full.html\#ref-list-1

License

Email Alerting

Receive free email alerts when new articles cite this article - sign up in the box at the top Service right corner of the article or click here.

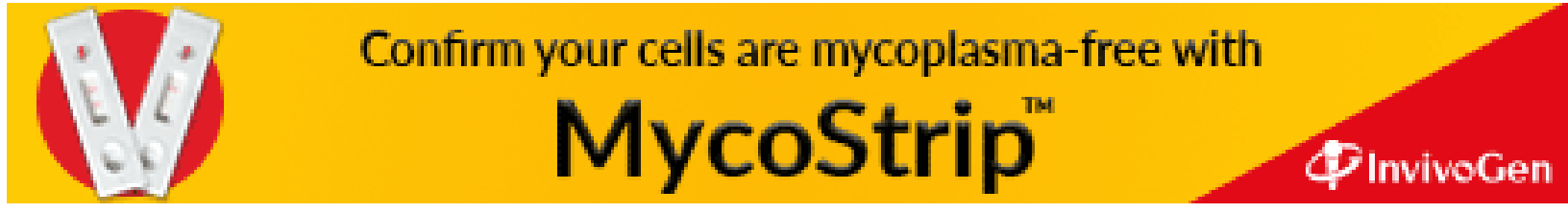

